\title{
Current Therapeutic Strategies of Parkinson's Disease
}

\author{
Poonkuzhali $\mathrm{K}$ and Jeevaratnam $\mathrm{K}^{*}$ \\ Department of Biochemistry and Molecular Biology, Pondicherry University, India
}

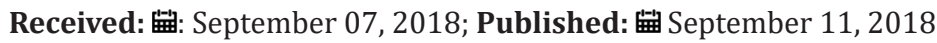

*Corresponding author: Jeevaratnam K, Department of Biochemistry and Molecular Biology, Pondicherry University, Kalapet, India

\section{Mini Review}

Parkinson's Disease (PD) is a chronic neurological disease which frequently encountered in the field of clinical medicine. The main motor symptoms are inflexibility, tremor, limb stiffness (akinesia) and instability to posture adding to its, changes in voice, freezing of gait, posture impairment and masking also occur and the non-motor symptoms might include cognitive impairment, autonomic nerve dysfunction, loss of interest and feel sad [1,2]. Various motor and non-motor PD symptoms have complicated the identification besides therapeutic challenges at present. Dopaminergic agents have been used for long term treatment might create the therapy to look forward for continuous recalibration, surgical therapy and further behavioural mechanism of pharmacological drug too in need [3]. For innovative therapeutic approaches, an effective neuroprotective therapy was developed and applied specifically intended for understand the interaction between neurodegeneration and aging, as well as it explained us to reassess the disease mechanism broad [4].

The animal models have been considered usually headed for test putative therapeutic agents and this was produced the problematic challenge to check the human diseases like PD in which replicate the clinical syndrome [5]. The target specific studies in patients are at risk and it rescued through the futuristic advances in brain imaging and molecular genetics possibly for the elucidation of PD starts and progresses [6]. Eventually, put an end to neurodegenerative process expect all the above concepts took as a first definitive step towards finding a cure. Levodopa is considered as safest medication for treatment of PD for past two decades. The levodopa used in early therapy created substantial treatment in doubt. Thereby, clinical trials have suggested that without specific reasons withhold early levodopa therapy was under risk [2]. The problem is, for few years' levodopa works well but then it starts to get these side effects like dyskinesia and uncontrollable movements. Scott S recently reported that ketamine's was having ability to offsetting dyskinesia when Parkinson's patients consumed it as a pain-relieving drug [7].

In combination of levodopa with ketamines were administered for individuals on trial, the unintended effect was observed as dyskinesia was ameliorated or disappeared for certain weeks. An additional common risk was blood pressure have been raised by using ketamine and it also used as a party drug [7]. Dopamine agonists (ropinirole and pramipexole) are also considered safe; nevertheless, there was increased in 2 to 3 -fold chances of raised impulse control disorders reduced prior interest for agonist initiation at early [8]. Monoamine Oxidase Inhibition has been carried out by its inhibitors (MAOIs) through the elevated levels of serotonin, norepinephrine and dopamine. Selegiline show small symptomatic effect on medication wearing off on early period of PD [9]. Newly MAO-B formulation was developed and named as 'rasagiline' by the movement disorders society in patients with placebo trial for evaluate the mechanism behind disease modification However, this drug also botched to reduce the disease development [10].

Levodopa in combination with carbidopa administration induced the motor fluctuations and dyskinesia. This difficult was addressed by altering the dosage of drug, regulating medication intervals and by added amantadine, MAO-B or a Catechol-O-Methyl Transferase Inhibitor (COMT) although dyskinesia was increased later. Entacapone a specific COMT inhibitor act as peripherally to improve motor fluctuations and Levodopa Induced Dyskinesia (LID) in PD [11]. Bromocriptine and pergolide was used as ergotbased dopamine agonists no longer period because of associated pulmonary fibrosis and heart valve problem. The principle behind the oral dopaminergic medications and disturbance of its absorption has been addressed by subcutaneous injections of apomorphine [12]. Recently, Nanoparticles (NPs) have been reported for easy drug delivery through Blood Brain Barrier (BBB) into the Central Nervous System (CNS) against conventional drug delivery systems $[13,14]$.

\section{Current Research Status on PD}

a. The transplantation of dopamine-producing neurons by grasped purified stem cells has auspiciously treats PD [15].

b. GPNMB (Glycoprotein non-metastatic melanoma protein B) protein got elevated and initiates the neurodegeneration in PD patients by modification in different types of fat in a brain area named the substantia nigra [16]. 
c. The clinical study has been defined that high levels of blood cholesterol in particularly Low-Density Lipoprotein Cholesterol (LDL-C) possibly reduced the threat of men developing PD [17].

d. The new-fangled study narrated that 'pain' was a PD marker before the appearance of motor disturbances [18].

e. The researchers have found that acrolein-a toxic byproduct of fat might promote the accumulation of alphasynuclein protein at substantia nigra and it leads to destroy the cell membranes and key machineries of neurons [19].

f. The drug, named NLY01, was tested on live mouse models and cultures of human brain cells, where it was observed to block brain cell degradation that results from Parkinson's disease [20].

g. The tears alpha synuclein protein level have been represent as a reliable biomarker for cheap mode of finding people with early stage of Parkinson's disease [21].

h. The new rat model was developed with increased alpha synuclein level in disorders of both PD dementia and dementia with Lewy bodies [22].

i. The Phase 2 clinical trial by Novartis exhibit nilotinib-a tyrosine kinase inhibitor have been modulated the dopamine levels and prevent the formation of toxic alpha-synuclein aggregates in patients with PD [23].

j. Fresh look on neurosurgical executes deep brain stimulation of Subthalamic Nucleus (STN) or Pedunculopontine Nucleus (PPN) to treat neurological conditions occur in PD and its mechanisms underlying the therapeutic effects remain unclear [24].

k. $\quad \mathrm{CeO}_{2}$ (cerium oxide) nanoparticle has been proposed as potential inhibitor of $\alpha$-synuclein and can be employed as nanodrug against the PD. Metal NPs such as GOLD NPs and SPIONs (superparamagnetic iron-oxide nanoparticles) also have the efficacy to inhibit the $\alpha$-synuclein [13].

l. GQDs (Graphene quantum dots) was used for in vivo penetration of NPs to blood brain barrier and protect against dopamine neuron loss induced by $\alpha$-syn preformed fibrils, Lewy body/Lewy neurite pathology [14].

As a conclusion, the results of newly identifying biomarkers on early therapeutics stage in animal models will develop the outcome for human disease. Although, the research on drug cure for PD have produced sole side effects. Let us comprehend the influence of existing dopaminergic drugs together with improvement of new pharmaceutical agents and new surgical approaches, to diminish the complications are of critical importance in the future treatment of PD. As a consequence, the enduring dopaminergic cells are rescued by futuristic novel tactics through ultimate prosper.

\section{References}

1. Díez Cirarda M, Ibarretxe Bilbao N, Peña J, Ojeda N (2018) Neurorehabilitation in parkinson's disease: A critical review of cognitive rehabilitation effects on cognition and brain. Hindawi Neural Plasticity 2018: 12 .

2. Okun MS (2017) Management of Parkison Disease in 2017: Personalized approaches for patient-specific needs. JAMA 318: 791-792.

3. Radhakrishnan DM, Goyal G (2018) Parkinson's disease: A review. Neurology India 66: 26-35.

4. Valera E, Masliah E (2016) Therapeutic approaches in parkison's disease and related disorders. J Neurochem 139: 346-352.

5. Obeso JA, Stamelou M, Goetz CG, Poewe W, Lang AE, et al. (2017) Past, present, and future of parkinson's disease: A special essay on the $200^{\text {th }}$ anniversary of the shaking palsy. Mov Disord 32: 1264-1310.

6. Nalls MA, Escott Price V, Williams NM, Lubbe S, Keller MF, et al. (2015) Genetic risk and age in Parkinson's disease: continuum not stratum. Mov Disord 30: 850-854.

7. Cohut M (2018) Ketamine for parkinson's? Clinical trial in the works. Medical News Today, Springer.

8. Grall Bronnec M, Victorri Vigneau C, Donnio Y, Leboucher J, Rousselet M, et al. (2018) Dopamine agonists and impulse control disorders: A complex association. Drug saf 41: 19-75.

9. Wolfgang H Oertel (2017) Recent advances in treating Parkinson's disease. F1000Res 6: 260.

10. Rachel D (2017) Generic drugs and parkinson's. FoxFeed Blog.

11. Lee Witt PA, Fahn S (2016) Levodopa therapy for Parkinson's disease: A look backward and forward. Neurology 86: S3-S12.

12. Seeman P (2015) Parkinson's disease treatment may cause impulsecontrol disorder via dopamine D3 receptors. Synapse 69:183-189.

13. Kaushik AC, Bharadwaj S, Kumar S, Dong Qing W (2018) Nanoparticle mediated inhibition of Parkinson's disease using computational biology approach. Scientific Reports 8: 9169.

14. Donghoon K, Je Min Y, Heehong H, Junghee L, Su Hyun L, et al. (2018) Graphene quantum dots prevent $\alpha$-synucleinopathy in Parkinson's disease. Nature Nanotechnol 13: 812-818.

15. Fathi A, Mirzaei M, Dolatyar B, Sharifitabar M, Bayat M, et al. (2018) Discovery of novel cell surface markers for purification of embryonic dopamine progenitors for transplantation in Parkinson's Disease animal models. Mol Cell Proteomics 17: 1670-1684.

16. Moloney EB, Moskites A, Ferrari EJ, Isacson O, Hallett PJ (2018) The glycoprotein GPNMB is selectively elevated in the substantia nigra of Parkinson's disease patients and increases after lysosomal stress. Neurobiol Dis 120: 1-11.

17. Rozani V, Gurevich T, Giladi N, El Ad B, Tsamir J, et al. (2018) Higher serum cholesterol and decreased Parkinson's disease risk: A statin-free cohort study. Mov Disord.

18. Acharya MK, Das S, Ganguly G, Das SK (2018) Pain: A marker of prodromal Parkinson's disease. World congress on Parkinson's disease and related disorders (IAPRD). Lyon, France, p. 19-22.

19. Ambaw A, Zheng L, Tambe MA, Strathearn KE, Acosta G, et al. (2018) Acrolein-mediated neuronal cell death and alpha-synuclein aggregation: Implications for Parkinson's disease. Mol Cell Neurosci 88: 70-82.

20. Yun SP, Kam TI, Panicker N, Kim S, Oh Y, et al. (2018) Block of A1 astrocyte conversion by microglia is neuroprotective in models of parkinson's disease. Nat Med 24: 931-938.

21. Feigenbaum D, Lew M, Janga S, Kunjeshkumar Shah M, Mack W, et al. (2018) Tear proteins as possible biomarkers for Parkinson's disease. Neurology 90: 19-22.

22. Espa E, Clemensson EK, Francardo V, Luk KC, Heuer A, et al. (2018) Cognitive and histopathological phenotypes in new rat models of cortical synucleinopathy. World congress on Parkinson's disease and related disorders (IAPRD). Lyon, France. 
23. Pagan F, Hebron M, Torres Yaghi Y, Lawler A, Kimbason T, et al. (2018) Nilotinib increases dopamine metabolism and reduces oligomeric: total alpha-synuclein ratio in Parkinson's disease. 19-22.

\section{ISSN: 2574-1241}

DOI: $10.26717 / B J S T R .2018 .08 .001729$

Jeevaratnam $K$. Biomed J Sci \& Tech Res

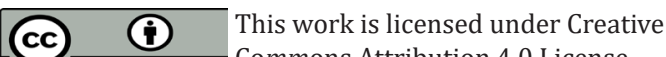

Submission Link: https://biomedres.us/submit-manuscript.php
24. Huang C, Chu H, Zhang Y, Wang X (2018) Deep brain stimulation to alleviate freezing of gait and cognitive dysfunction in Parkinson's disease: Update on current research and future perspectives. Front Neurosci 12: 29.

Assets of Publishing with us
- Global archiving of articles
- Immediate, unrestricted online access
- Righes

